\title{
Classes sociais e simbolização na cidade do Porto Elementos teóricos e resultados de pesquisa empírica
}

\author{
Virgílio Borges Pereira
}

Introdução

No presente texto procuramos demonstrar que a obra sociológica de Pierre Bourdieu constitui um referencial heurístico significativo para o desenvolvimento de investigação sociológica sobre as divisões de classe e sobre os processos de simbolização na cidade. Tomando por referência o trabalho sociológico de Bourdieu em La distinction, bem como o conhecimento das relações entre a produção das divisões sociais e a produção das divisões simbólicas sugerido nessa e noutras obras que marcaram o percurso intelectual do sociólogo francês, procuraremos explicitar as coordenadas teóricas e metodológicas fundamentais que estiveram subjacentes ao desenvolvimento continuado de uma pesquisa sociológica sobre classes sociais e processos de simbolização na cidade do Porto, no norte de Portugal. Para além de documentar o diálogo que foi possível estabelecer entre o corpo de proposições teóricas e metodológicas decorrentes do trabalho de Bourdieu e os desenvolvimentos da sociologia das classes sociais praticada em Portugal, o texto explica, sinteticamente, o percurso teórico-empírico formulado na análise da cidade do Porto entre o final da década de 1990 e o início dos anos 2000, terminando com uma breve discussão da análise efetuada, mais recentemente, em um dos bairros da cidade. Procura-se, com este procedimento, não só ressalvar a importância de um questionamento relacional sobre os processos de constituição das divisões sociais e simbólicas na cidade, mas também, e por essa via, demonstrar o potencial teórico e metodológico contido no programa de pesquisa assim estabelecido. 
Analisar classes sociais e processos de simbolização a partir do legado sociológico de Pierre Bourdieu

$\mathrm{Na}$ sequência do seu desembarque argelino, é sabido que Pierre Bourdieu desenvolveu um intenso trabalho de conversão do olhar que o conduziria, progressivamente, a uma articulação sistemática de teoria e investigação empírica de matriz antropológica e sociológica que muito contribuiria para reorientar, ao longo dos anos que se seguiriam, o caminho da sociologia contemporânea (Wacquant, 2004; Martin-Criado, 2008). Nessa reorientação, em que alguns autores verão, mais tarde, propriedades copernicianas (L. Pinto, 1998), Bourdieu pôde efetuar uma síntese criativa e rigorosa de diferentes tradições teóricas de estudo do poder e da dominação, inscrevendo as divisões sociais no centro das suas preocupações de pesquisa e contribuindo, desse modo, para reformular amplamente os caminhos da análise sociológica das classes sociais (Wacquant, 2013).

Anunciada e teorizada nos seus diferentes estudos argelinos (Bourdieu et al., 1963; Bourdieu e Sayad, 1964; Bourdieu, 1977) e prolongada nas pesquisas que desenvolveria sobre a França dos anos de 1960 (Bourdieu, 1966), essa preocupação com a gênese e a estruturação das classes sociais seria, desde muito cedo, também acompanhada por uma interrogação sobre as relações que se estabelecem entre estas e as divisões simbólicas, ao abrigo de um estudo sistemático sobre os processos de socialização escolar e cultural (Bourdieu, [1978] 2013). Sem que o trabalho desenvolvido estivesse necessariamente ao serviço de um propósito de teorização pré-estabelecido, na sequência da publicação de "L'anatomie du gôut" (Bourdieu e Saint-Martin, 1976), Bourdieu estabiliza em La distinction o procedimento analítico subjacente a este exercício, argumentando, com base em extensa reflexão teórica solidária de um ambicioso trabalho empírico, sobre a relevância de uma concepção efetivamente relacional das classes sociais e sobre a importância dos processos de divisão simbólica na sua estruturação (Bourdieu, 1979; 2011).

A sociologia das classes sociais e dos processos de divisão simbólica assim perspectivada passa a ter um repertório de procedimentos preciso, que Bourdieu parece nunca ter deixado de tentar aperfeiçoar ao longo do tempo, se levarmos em conta os exercícios de reescrita a que vários dos seus textos sobre esta matéria estiveram sujeitos e os próprios propósitos de regresso à temática, para efeitos de sistematização teórica, que deixou anotado em passagens determinadas de La distinction e que nunca foi concretizado nos termos nesta previstos ${ }^{1}$. A sociologia aqui em causa conjugará, assim, a dinamização de inquéritos sobre práticas, classificações e pertenças sociais

1. Sobre este assunto, ver Bourdieu (1979, p. 128, nota 23). 
com trabalho etnográfico de perfil diferenciado e o desenvolvimento de leituras relacionais da informação recolhida, acrescida da mobilização da técnica estatística de análise de correspondências múltiplas (Rouanet et al., 2000; Benzécri, 1992). Como se depreende do estabelecido, a análise empreendida por Bourdieu convoca classes e frações de classe, mas o faz potenciando relações entre diferentes propriedades sociais e acentuando a importância de uma leitura, configurada em termos topológicos sob a forma de espaço social, das divisões socialmente construídas em torno do volume e da estrutura da composição de capital, com particular destaque para o econômico e para o cultural, e sobre a importância da sua evolução no tempo (Bourdieu, 1979, pp. 128-144). Suportado em uma visão tripartida do espaço social, diferenciado em zonas dominantes, intermediárias e dominadas, Bourdieu considera também pertinente proceder a um retrato topológico homólogo, sob a forma de espaço dos estilos de vida, dos processos de simbolização, marcados pelo conhecimento e pelo reconhecimento de sentido em torno da "distinção", da "tensão" e do "necessário" estabelecidos pelos agentes sociais através de processos que qualificou, sob inspiração weberiana, como de "estilização da vida" (Idem, pp. 59-61, 195, 198). Idênticas consequências são retiradas do ponto de vista assim esboçado no plano político, para além daquelas que dizem respeito ao modo de estruturação das divisões simbólicas no interior de cada uma das três grandes regiões do espaço social. Se, no plano político, as divisões em causa passam pela relevância das oposições entre a esquerda e a direita e sobre os fundamentos sociais das variações estabelecidas em torno dos quadrantes políticos em questão (Idem, pp. 512-527), no que diz respeito às divisões simbólicas configuradas no interior das três grandes regiốes do espaço social, Bourdieu identifica quadros de divisão social e de luta simbólica significativos, seja entre os que ocupam regiões dominantes - desde logo, os que dependem da composição relativa do capital econômico e cultural e da oposição entre gostos burgueses e gostos intelectuais -, seja ainda entre os que ocupam as regiões intermediárias e dominadas e as dinâmicas de (re)produção do gosto sob tensão e de necessidade que suportam (Idem, capítulos 5-7).

Por outro lado - e ainda que não seja uma proposta teórica imediatamente associada à conceitualização da cidade contemporânea -, o trabalho sociológico de Pierre Bourdieu em La distinction tem condições para ser mobilizado criativamente para conhecer os processos de estruturação desta. De resto, Bourdieu dava nesta obra indicações significativas de que o espaço físico estava muito longe de ser socialmente indeterminado e de que haveria grande interesse em integrá-lo ao ponto de vista topológico por si defendido para ler a realidade social:

[...] uma classe ou uma fração de classe é definida não somente pela sua posição nas relações de produção tal como pode ser captada através de índices como a profissão, os rendimentos, 
ou mesmo o nível de instrução, mas também por uma certa proporção sexual, uma distribuição determinada no espaço geográfico (que nunca é neutro socialmente) e por todo um conjunto de características auxiliares que, a título de exigências tácitas, podem funcionar como princípios de seleção ou de exclusão reais sem nunca serem formalmente enunciados [...] (Idem, p. 113, tradução minha)

Em investigações posteriores, Bourdieu fornecerá elementos analíticos adicionais que permitirão documentar processos de autonomização de capitais específicos e identificar os quadros de relações que os suportam nas regiões mais favorecidas do espaço social, abrindo novas linhas de investigação muito significativas nos domínios do campo do poder e da educação (Bourdieu, 1989), mas também em diferentes regiões do campo cultural e artístico (Bourdieu, 1992), para além dos que dizem respeito à análise da produção e da reprodução do metacapital decorrente da ação do Estado (Bourdieu, 1993a) e da ação deste na economia (Bourdieu, 2000), entre outros aspectos. Por outro lado, e como também sabido, a atenção consagrada à estruturação de campos e capitais específicos não deixou de ser articulada sistematicamente com o interesse pelo conhecimento da experiência dos agentes sociais, bem como dos processos de formação das respectivas competências práticas e simbólicas sobre a prática, seja dos que protagonizam tais processos de autonomização de capitais e inscrevem os seus posicionamentos, mais ou menos estabilizados, nos campos em questão, seja também os daqueles que, nas regiões mais desfavorecidas do espaço social, vivenciam quadros de sofrimento social (Bourdieu, 1993b). Numa linha de pensamento que conjugava o legado de Marx e de Weber com o de Durkheim e o da tradição fenomenológica, a proposta teórica de Bourdieu, alimentada por múltiplas frentes de pesquisa, apurava um movimento de análise que articularia um propósito de conhecimento da relação entre a força das coisas e as modalidades da sua incorporação. Tal como teve ocasião de sistematizar, para Bourdieu, o princípio da ação:

[...] reside na cumplicidade entre dois estados do social, entre a história feita corpo e a história feita coisa, ou, mais precisamente, entre a história objetivada nas coisas, sob a forma de estruturas e de mecanismos (os do espaço social ou dos campos), e a história encarnada nos corpos, sob a forma de habitus, cumplicidade que funda uma relação de participação quase mágica entre estas duas realizações da história (Bourdieu, 1997, p. 179 , tradução minha).

Ainda que La distinction seja uma obra muito discutida, não são frequentes os exercícios que procuraram dialogar, a partir de referenciais teórico-empíricos mais 
diretos e comparáveis, com o referido legado ${ }^{2}$. Não obstante a relativa complexidade das múltiplas implicações teóricas e metodológicas subjacentes aos procedimentos nele contidos, o legado teórico decorrente da obra de Bourdieu - e, especificamente, o mais diretamente dependente de La distinction - pode ser assumido com clareza no desenvolvimento de pesquisa sociológica de diferentes âmbitos sociais e territoriais. Pesquisas conduzidas na Noruega (Rosenlund, 2009), na Dinamarca (Prieur et al., 2008) e no Reino Unido (Bennett et al., 2008), entre outras, permitiram, mais recentemente, construir esse diálogo a partir de referenciais mais controlados. Vejamos o que pode ser dito a este propósito tomando por referência investigação desenvolvida na cidade do Porto.

\section{Classes sociais e processos de simbolização na cidade do Porto contemporânea}

Como se depreende do que temos vindo a sustentar, as coordenadas teóricas, o perfil de trabalho metodológico e a lógica geral dos resultados decorrentes da investigação de Pierre Bourdieu foram-se afirmando nas nossas preocupações como suscetíveis de se constituírem em um referencial heurístico para conhecer a cidade contemporânea. À luz dessas preocupações, desde cerca de meados dos anos de 1990 que tomamos a cidade do Porto, no norte de Portugal, como contexto de referência para um questionamento das relações sociais informado pelo programa de pesquisa proposto em La distinction. No centro da abordagem, colocavam-se-nos três grandes domínios autônomos, mas interrelacionados, de análise. $\mathrm{O}$ primeiro dizia respeito à compreensão dos processos de formação das divisões de classe na sociedade portuguesa e da sua importância específica para a leitura sociológica da cidade do Porto. O segundo domínio procurava conhecer os processos de estruturação das vivências quotidianas dos residentes da cidade e, nesse sentido, abria os propósitos de trabalho sociológico ao conhecimento das práticas sociais e culturais dos residentes, a partir de uma análise detalhada dos usos do tempo e das representações dos agentes sociais com objetivos de leitura das dinâmicas de causalidade relacional subjacentes à sua produção e ao quadro de divisões sociais em que estas se inscreviam. Acrescentamos ainda a este programa um terceiro ponto de vista que procurava inscrever a análise no quotidiano dos diferentes contextos espaciais da cidade e que tinha implicações significativas no desenho metodológico da pesquisa.

O propósito de análise dos processos de formação de classe implicou o desenho de um quadro de questões fundado na tradição de análise sociológica das classes sociais construída em Portugal, nomeadamente através dos estudos que José Madureira

2. Para uma síntese de questionamentos e de respostas sobre a recepção de La distinction, ver Duval (2010). 
Pinto (1985) e João Ferreira de Almeida (1986) desenvolveram sobre a matéria e da mobilização do esquema de análise de classes sociais tripartido inspirado no trabalho desses autores (Almeida et al., 1988; 2006), sendo tal modelo originalmente marcado, com base em uma conjugação de heranças marxistas e weberianas, pelas próprias propostas de análise sociológica de Bourdieu sobre classes sociais.

O ponto de vista teórico formulado para compreender práticas sociais e divisões simbólicas implicou a dinamização de uma teoria específica sobre a estruturação do tempo, que procurou inspiração nos estudos de Pierre Bourdieu (1977), Norbert Elias e Eric Dunning (1992), Marcel Maget (1962) e em vários analistas das temporalidades sociais (Lalive d'Épinay et al., 1982; Donnat, 1998, entre outros). A relação com domínios de prática significativos, como os da religião, da política ou da sociabilidade local, foi complementada com o estudo de processos de classificação e de representação social, inspirados em contributos variados que retinham, desde logo, resultados de La distinction.

Com base nos marcos analíticos previamente explicitados, foi possível desenvolver uma estratégia de objetivação que apelou à formulação de um quadro de compreensão sociológica capaz de conjugar abordagens extensivas e etnográficas, inspirando-se não só nos próprios exemplos de pesquisa de Bourdieu (1979; 2003), mas também nas perspectivas dinamizadas por outros sociólogos, como Michael Burawoy (1998), Michel Bozon (1984) ou Loïc Wacquant (2008), fazendo do conhecimento prévio e detalhado do tecido social local da cidade uma preocupação fundamental.

Elaborou-se, assim, uma estratégia de objetivação que apelou à combinação de métodos de pesquisa alternativos e à conjugação de diferentes modos de construção do olhar sociológico sobre a cidade. Reconstituíram-se, em um primeiro momento, estatísticas oficiais sobre a história regional e local dos processos de (re)produção de desigualdades (laborais, socioprofissionais, escolares), o que permitiu definir os perfis das divisões sociais que estruturavam o território citadino e seu entorno. Desenvolveu-se, em um segundo momento, ocorrido durante o ano 2000, um extenso inquérito por questionário sobre classes sociais e estilos de vida a 1.043 grupos domésticos repartidos em dezoito contextos territoriais representativos da cidade. Entre os anos 2000 e 2001, dinamizou-se, em um terceiro momento, articulado com o anterior, um programa de observação etnográfica de sete contextos habitacionais e realizou-se um conjunto de entrevistas semidiretivas com mais de cem agentes que viviam e/ou trabalhavam nos mesmos contextos (Pereira, 2005, pp. 801-807).

Uma revisitação, muito sintética, dos resultados reunidos neste trabalho permite aferir a pertinência do enquadramento teórico-metodológico proposto e das opções formuladas nos diferentes planos de pesquisa para conhecer em profundidade as dinâmicas de formação das divisões sociais e simbólicas na cidade. 
Habitualmente considerada a segunda cidade de Portugal, a cidade do Porto é o mais importante centro urbano do norte de Portugal e a referência da área metropolitana a que dá nome. No início dos anos 2000, a população da cidade intramuros era pouco superior a 260 mil habitantes, encontrando-se o seu ritmo de crescimento em clara perda. A reconstituição da história recente da estrutura de classes na cidade e na região metropolitana resultou na identificação de propriedades específicas da cidade do Porto por comparação com os municípios circundantes, permitindo destacar o relevo da economia dos serviços na definição do emprego na cidade e uma diminuição significativa do emprego operário industrial, assim como uma concentração significativa, por comparação com o restante contexto metropolitano, de profissionais mais qualificados em capitais econômicos e culturais (Idem, pp. 61-103).

A informação apresentada na Tabela 1 documenta, por sua vez, os resultados obtidos com o inquérito efetuado na cidade do Porto em 2000 e regista a pertença de classe familiar geral dos membros das famílias inquiridas. A fração de classe modal, numa tendência equivalente à sociedade portuguesa em geral ${ }^{3}$, é constituída pelos empregados de execução. As pertenças operárias seguem-se em ordem de importância. As restantes frações de classe apresentam efetivos mais restritos, embora todas elas com presença agregada não inferior a 10\%. Em todo caso, tanto os agentes pertencentes às frações independentes e de pequenos proprietários como os intelectuais apresentam efetivos ligeiramente mais densos do que os relativos aos profissionais técnicos e de enquadramento intermediário e aos empresários, dirigentes e profissionais liberais.

\begin{tabular}{l|c} 
TABELA 1 & \\
Individuos Recenseados no Estudo sobre Classes e Estilos de Vida na \\
Cidade do Porto Segundo a Situação de Classe da Família, ano 2000 \\
\hline Empresários, dirigentes e profissionais liberais & $10 \%$ \\
Intelectuais & $13 \%$ \\
Profissionais técnicos e de enquadramento intermediário & $10 \%$ \\
Independentes e pequenos proprietários & $13 \%$ \\
Empregados de execução & $30 \%$ \\
Operários & $25 \%$ \\
\hline Total & $100 \%(\mathrm{n}=3.081)$ \\
\hline
\end{tabular}

Fonte: Inquérito Porto - Classes e Estilos de Vida, 2000 (Pereira, 2005).

3. Para um retrato comparativo recente da estrutura de classes portuguesa, ver Almeida et al. (2006). 
Tal como previsto, a análise promovida sobre a informação recolhida no inquérito procurou complementar informação sobre as classes e as frações de classe dos inquiridos com outra informação pertinente, relativa a formas diferenciadas de capital econômico, cultural e social, para além da que dizia respeito ao próprio contexto citadino de residência. A análise relacional dessa informação, efetuada com recurso à técnica de análise de correspondências múltiplas nos termos sugeridos pelo trabalho de Bourdieu e por alguns dos mais recentes desenvolvimentos metodológicos em torno do seu manuseamento (Le Roux e Rouanet, 2004; 2010; Lebart et al., 1998), permitiu definir a configuração do espaço social citadino. Neste, foi possível demarcar, em um primeiro eixo, uma polarização muito significativa em termos de volume global de capital, solidária de uma divisão entre os agentes com pertença e origem nas classes e frações mais qualificadas nas diferentes formas de capital e aqueles que, com pertença e origem sobretudo operária, revelavam maior fragilidade nos capitais sob observação. Por sua vez, em um segundo eixo, era igualmente possível documentar um efeito de composição do capital, com a identificação de uma divisão significativa entre agentes mais dotados, em termos relativos, em capital econômico e agentes mais definidos em torno de posicionamentos sociais estabelecidos acerca do capital cultural (Pereira, 2013, pp. 210-211 $)^{4}$. A utilização do espaço social assim definido como mapa preditivo de práticas e de representações (Lebart et al., 1984, p. 100) permitiu, por sua vez, definir relações estatísticas e sociologicamente significativas entre os posicionamentos no espaço social identificados e os processos de simbolização que os caracterizavam, documentando-se, entre os agentes que ocupavam as regiões do espaço social citadino definidas em torno do maior volume de capital, práticas de sociabilidade e práticas culturais mais diversificadas e mais intensamente desenvolvidas, em contraste com práticas menos diversificadas e, com algumas exceções sociologicamente significativas, bem menos intensas que caracterizavam aqueles que ocupavam as regióes mais desfavorecidas. A divisão decorrente da composição estrutural dos capitais dos agentes observada no segundo eixo do espaço social era igualmente caracterizada por relações pertinentes aos domínios da sociabilidade e das práticas culturais estudados, documentando-se entre os posicionamentos dos empresários traços de uma sociabilidade mais intensa e, entre intelectuais, registros de sociabilidade mais disciplinada e de práticas culturais mais eruditas (Pereira, 2013, p. 211).

Em articulação com esse procedimento, e não obstante as conclusões já estabelecidas, procuramos aprofundar o exercício de construção da relação de homologia entre o espaço social e o universo das possíveis estilísticas dos inquiridos. Para além de vários

4. Informações mais pormenorizadas sobre os problemas em apreço podem também ser obtidas em Pereira (2005, pp.113 e ss). 
exercícios de análise relacional que permitiram estudar as práticas de ocupação do tempo em função de quadros espaciais mais delimitados (domésticos, locais, urbanos) (Pereira, 2005, capítulo 4), foi também possível construir uma proposta interpretativa geral sobre a lógica de estruturação das divisões simbólicas na cidade, lendo autonomamente a informação sobre práticas e gostos e submetendo-a a idêntico procedimento de análise de correspondências múltiplas. O exercício em causa - construído com recurso a 33 variáveis relativas a práticas e gostos televisivos, de leitura, de escuta musical, de atividades culturais, de sociabilidade e atividade desportiva - permitiu definir o espaço dos estilos de vida dos agentes sociais inquiridos na cidade do Porto. Neste, foi também possível revelar, por um lado, modalidades significativamente diferenciadas de estruturação do gosto, da sociabilidade e da cultura. Desde logo e em um primeiro eixo, gostos definidos em torno da forte participação cultural e sociabilitária de pendor seletivo e, ao contrário, gostos definidos em torno de fraca participação e mais populares; em alternativa, e em um segundo eixo, foram identificados gostos de configuração mais comercial em oposição a gostos intelectuais. Por outro lado, foi igualmente demonstrado que os processos de estruturação das práticas conceitualizadas não eram produto de uma eventual indeterminação social, não estando, por isso, a sua produção isenta de causalidade social com significado sociológico: a primeira divisão do espaço dos estilos de vida era caracterizada por uma forte oposição em termos de volume global de capital; a segunda divisão identificada era caracterizada por uma oposição relevante em termos passíveis de serem lidos ao abrigo de uma oposição sensível aos efeitos da composição dos capitais econômicos e culturais dos agentes inquiridos (Pereira, 2015).

A construção do espaço social e do espaço dos estilos de vida portuenses foi também complementada com um trabalho de análise sobre os processos de produção das práticas políticas e ideológicas dos inquiridos. Esse trabalho conduziu à elaboração de uma leitura das relações entre o espaço social e o espaço de tomadas de posição simbólico-ideológica. Para além de configurações de sentido congruentes com o que se conhece sobre os processos analisados na sociedade portuguesa, o estudo da gênese social encontrou contrapartidas equivalentes às que acabamos de apresentar para os outros domínios de análise privilegiados, creditando o exercício de pesquisa produzido (Pereira, 2005, pp. 458-478).

Vejamos, agora, algumas incidências dos problemas aqui em discussão a partir de um ponto de vista construído sobre os bairros da cidade do Porto.

O espaço social e os processos de simbolização a partir do território vivido da cidade

Como tínhamos assinalado inicialmente, o trabalho de pesquisa conduzido sobre os processos de estruturação das classes sociais e dos estilos de vida dos habitantes 
da cidade do Porto realizado nos anos iniciais da década de 2000 foi elaborado em um quadro fortemente vinculado ao território citadino e com propósitos de conhecimento da tessitura do seu quotidiano. Tirava-se partido do conhecimento acumulado sobre a relevância das divisões sociais de classe, mesmo se de modo não linear, na estruturação da geografia social da cidade (Marques et al., 1990). Nesse sentido, e uma vez construído o espaço social da cidade, foi possível identificar relações significativas entre as lógicas que o constituíam e o espaço físico nestas inquirido, lido através de informação sobre os contextos de observação territorial projetada em termos suplementares nos eixos previamente descritos (Pereira, 2016). Do ponto de vista da estratégia geral da pesquisa, o conhecimento dessas relações permitiu aprofundar o trabalho metodológico e uma abertura a frentes de análise que procuravam articular o trabalho decorrente do inquérito sociológico e do seu tratamento relacional, com dados provenientes de pesquisa etnográfica e sócio-histórica situada em múltiplos contextos da cidade. Foi possível documentar, assim, margens de variação na estruturação em contexto do espaço social, do espaço dos estilos de vida e do espaço das tomadas de posição simbólico-ideológicas e definir, a partir de uma análise que consagrava o ponto de vista dos agentes sociais, "efeitos de lugar" social e historicamente determinados (Bourdieu, 1993c): as pesquisas conduzidas no centro histórico medieval da cidade permitiam revelar a importância da relação entre fragilização social e habitacional na debilitação da sociabilidade local (Pereira, 2005, pp. 483-558; Pereira e Pinto, 2012); a análise desenvolvida na área central de expansão oitocentista dava conta dos contrastes sociais e simbólicos envolvidos na produção da vida quotidiana urbana de intelectuais, de técnicos de enquadramento intermediário e de empregados de execução (Pereira, 2005, pp. 559-620); o estudo realizado num grande bairro de habitação social da periferia ocidental permitia revelar as diferenças sociais que se estruturavam ali e as relações que contemplavam complexos processos de estigmatização territorial (Pereira, 2005, pp. 621-695; Pereira e Queirós, 2014); a análise conduzida na faixa atlântica da cidade, montra da cidade burguesa, revelava, em múltiplos planos, a grande importância das divisões sociais e simbólicas que se configuravam no seu interior, não só as que se estabeleciam entre ricos e pobres, mas também as contradições e tensões que envolviam a antiguidade do privilégio cultural e econômico de quem vivia naquele lugar (Pereira, 2005, pp. 697-764).

O esforço de conhecimento assim empreendido suscitou a necessidade de aprofundamentos suplementares e, no quadro de um trabalho sobre as relações entre a ação do Estado e a constituição dos lugares da cidade, mais recentemente, pudemos repetir e aprofundar o programa de pesquisa que conjugava trabalho de inquérito, pesquisa etnográfica e análise sócio-histórica proposto nas análises previamente 


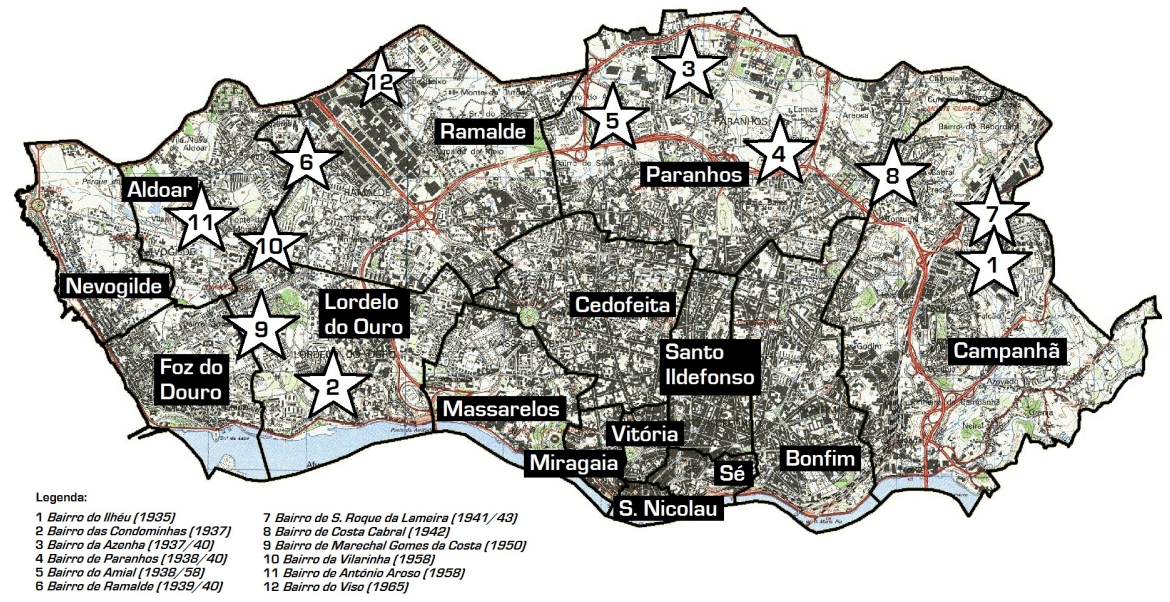

Fonte: PTDC/SDE/69996/2006.

citadas 5 . Para efeito de discussão ilustrativa sobre as dinâmicas constitutivas dos efeitos de lugar na cidade, destacamos aqui os resultados de um estudo conduzido, durante o ano de 2009, no Bairro do Amial. Espaço com 390 habitações em moradias individuais localizado no norte da cidade do Porto, o Bairro do Amial é fruto da iniciativa do Estado português e foi o maior dos bairros do Programa das Casas Econômicas erigido na cidade, com construções efetuadas nas décadas de 1930 e 1950 (Figura 1). Resultado de uma política traçada durante os anos iniciais do Estado Novo (1933) e que vigorou até 1965, o programa promoveu a construção de habitações novas em um quadro de crise habitacional e, ainda que suas realizações tenham ficado claramente aquém das necessidades, foi um elemento ativo no processo de urbanização da cidade a partir dos referenciais da "cidade-jardim", tendo resultado na promoção do acesso à propriedade habitacional em diferentes tipos de casa individual dos segmentos mais estabilizados do operariado e, sobretudo, da pequena-burguesia, constituindo a referência central das políticas habitacionais públicas durante a vigência do Estado Novo ${ }^{6}$.

5. Projeto "Ilhas, bairros e classes laboriosas: um retrato comparado da génese e estruturação das intervenções habitacionais do Estado na cidade do Porto e das suas consequências sociais”, coordenado pelo autor, financiado pela Fundação para a Ciência e Tecnologia (referência PTDC/sDE/69996/2006) e desenvolvido no Instituto de Sociologia da Universidade do Porto.

6. Para desenvolvimentos suplementares sobre os objetivos políticos subjacentes ao programa em análise e sobre a gênese do bairro em questão, ver Pereira e Queirós (2013); sobre a relevância das políticas habitacionais na definição do espaço físico e social da cidade, ver Pereira e Queirós (2012a). 
A informação obtida através do inquérito realizado localmente em 2009 reúne elementos representativos sobre 272 famílias $^{7}$. Para além da documentação exaustiva das características sociais de todos os residentes em cada uma das famílias inquiridas, a pesquisa permitiu obter informação sobre práticas e classificações sociais dos representantes de cada grupo doméstico ${ }^{8}$. A informação contida na Tabela 2 documenta as pertenças de classe dos residentes, captadas a partir de indicadores que retêm o grupo doméstico como unidade de análise. A população geral do Bairro do Amial (no momento do inquérito com um efetivo menor do que aquele que possuiu noutras fases $\mathrm{da}$ sua história) apresenta uma composição de classe dotada de alguma diversidade, mas destaca-se sobretudo pela presença de intelectuais, a que se seguem os empregados de execução e os técnicos de enquadramento intermediário. As restantes frações de classe têm presenças menos densas, ainda que não irrelevantes. Em todo caso, tanto no que diz respeito à leitura global da sua história social como do ponto de vista mais restrito da composição da população ativa, o bairro, não obstante a diminuição da população e o abandono de um segmento específico do edificado (nomeadamente das habitações menores), apresenta uma tendência para a qualificação dos capitais econômicos e culturais dos respectivos residentes, o que o diferencia significativamente da cidade modal e dos contextos residenciais envolventes.

TABELA 2

Fraçôes de Classe da Familia dos Ativos e da População Residente no Bairro do Amial (2009)

\begin{tabular}{l|c|c}
\hline Frações de Classe da Família & $\begin{array}{c}\text { Ativos Recenseados } \\
(\%)\end{array}$ & $\begin{array}{c}\text { População Recenseada } \\
(\%)\end{array}$ \\
\hline Empresários, dirigentes e profissionais & 11,0 & 10,2 \\
Intelectuais e cientistas & 28,1 & 23,6 \\
Técnicos e agentes de enquadramento intermediário & 16,8 & 18,4 \\
Independentes e pequenos proprietários & 16,4 & 14,6 \\
Empregados de execução & 20,4 & 21,3 \\
Operários & 7,5 & 11,9 \\
\hline Total & 100 & 100 \\
\hline
\end{tabular}

Fonte: PTDC/SDE/69996/2006, Inquérito IB 18, 2009.

7. Cerca de 65 habitações encontram-se desocupadas; outras 55 não responderam às várias tentativas que fizemos para obter informações a seu propósito.

8. Por razões relacionadas com o espaço disponível, não desenvolveremos as implicações sócio-históricas e mais orientadamente etnográficas do exercício de análise proposto. Estas podem ser aferidas com mais detalhe em Pereira e Queirós (2012b). 

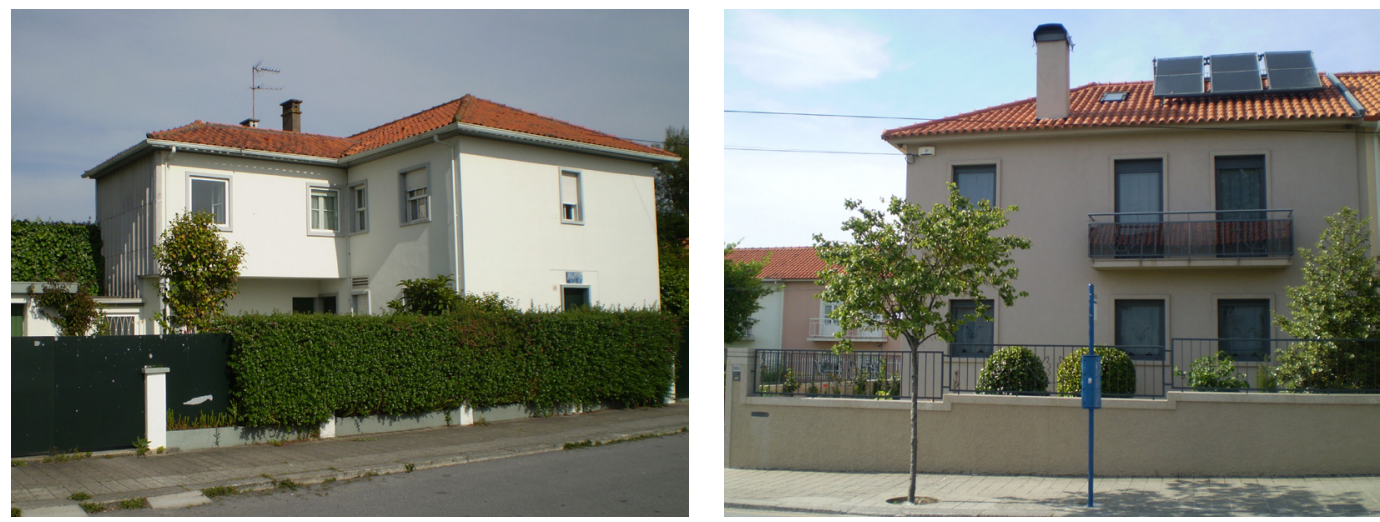

Fonte: João Queirós e PTDC/SDE/69996/2006.

Tal como previsto, procuramos construir uma visão relacional do processo de estruturação do espaço social local, e, nesse sentido, a informação sobre a composição de classe dos 272 grupos domésticos inquiridos foi complementada com elementos adicionais, levando-se em conta informação equivalente à que tinha sido reunida para construir o espaço social da cidade previamente apresentado (com a única exceção da variável relativa à localização no espaço geográfico da cidade) e, tal como se pode verificar na Tabela 3, a organização de quatro grandes domínios, a saber: classes sociais, capital econômico, capital cultural e capital social. A submissão da informação reunida a um procedimento de análise de correspondências múltiplas ${ }^{9}$ permite destacar, para efeitos interpretativos, os resultados dos seus dois primeiros eixos, que correspondem a um total de $78,4 \%$ de variância explicada, conforme se pode verificar na Tabela 4 (taxas modificadas).

As informações representadas no Gráfico 1 mostram as modalidades que mais contribuem para o primeiro eixo do espaço social local, que envolve a produção de divisões e posicionamentos sociais muito claros. No lado direito do gráfico é possível identificar associações entre os detentores de capitais culturais mais elevados, pertenças e origens de classe intelectuais, bem como as modalidades de capital econômico e social mais densas (possuir dois automóveis ou mais, usufruir habitualmente de férias fora de casa, ou de uma segunda residência; possuir ligação com internet e redes relacionais compostas por mais de cem pessoas). Por sua vez, no lado esquerdo da mesma figura é possível identificar um quadro de associações alternativas e que

9. Tal como com todos os exercícios anteriormente apresentados, foi efetuada uma análise de correspondências múltiplas "específica" com recurso ao programa SPAD, versão 7.4. 
TABELA 3

Dominios Sociais e Variáveis Utilizadas na Construção do Espaço Social do Bairro do Amial e Respectivo Número de Modalidades

\begin{tabular}{l|c}
\hline MODALIDADES & NúMERO DE MODALIDADES \\
\hline CLASSE & 7 \\
Origem de classe do grupo doméstico & 6 \\
Fração de classe do grupo doméstico de pertença & 13 \\
Total & \\
\hline CAPITAL EconômICo & 2 \\
Propriedade da casa & 2 \\
Fase de construção da casa no bairro & 2 \\
Posse de outra residência & 3 \\
Número de automóveis na família & 2 \\
Férias fora de casa & 11 \\
Total & \\
\hline CAPITAL CulTuraL & 5 \\
Capital escolar (em anos de escolaridade) & 6 \\
Número de livros que possui em casa & 11 \\
Total & \\
\hline CAPITAL Social & 5 \\
Densidade da rede relacional & 3 \\
Envolvimento associativo (prático) & 2 \\
Acesso à internet & 10 \\
Total & \\
\hline
\end{tabular}

TABELA 4

Análise de Correspondências Múltiplas Efetuada no Ambito da Pesquisa no Bairro do Amial: Eixos, Valores Próprios, Porcentagem de Variância Explicada e Porcentagem de Variância Explicada Modificada

\begin{tabular}{l|c|c|c|c}
\hline \multicolumn{2}{c}{ VARiÂNCIA TOtAL: 2.77246} \\
\hline Eixo & Valor Próprio & $\%$ & \% Modificada & \% Modificada acumulada \\
\hline 1 & 0,2900 & 10,46 & 71,8 & 71,8 \\
2 & 0,1399 & 5,05 & 6,6 & 78,4 \\
3 & 0,1322 & 4,77 & 5,1 & 83,5 \\
\hline
\end{tabular}

Fonte: PTDC/SDE/69996/2006, Inquérito IB 18, 2009 (tratamento através de ACM específica).

relevam o vínculo entre os agentes que se definem por pertenças e origens operárias e pela posse de modalidades menos favorecidas de capital cultural, econômico e social (quatro anos de escolaridade, menos de vinte livros em casa; não usufruir de férias fora de casa, não possuir automóvel, ausência de propriedade habitacional; não 
GRÁFICO 1

Espaço Social do Bairro do Amial (Dezoito Modalidades Ativas no Eixo 1)

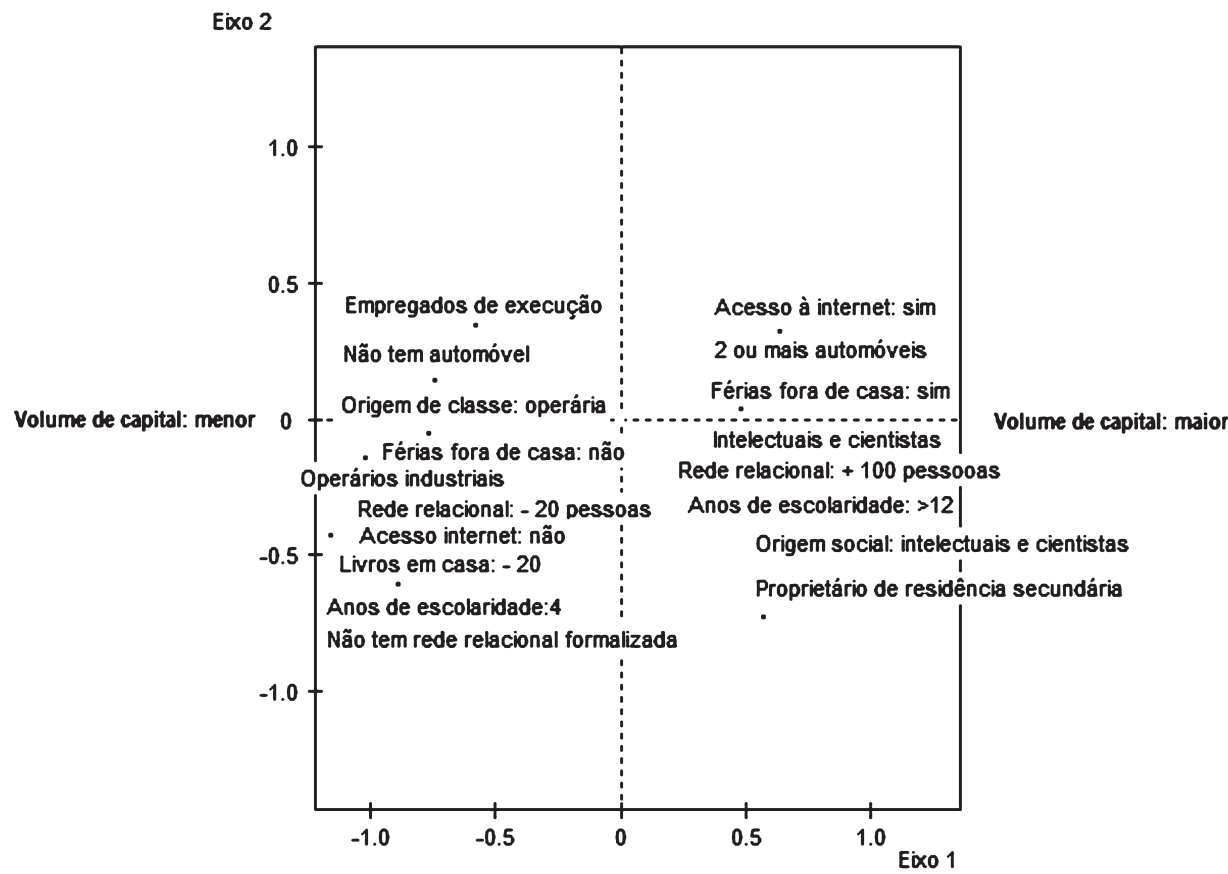

Fonte: PTDC/SDE/69996/2006, Inquérito IB18, 2009.

possuir ligação à internet, não ter agenda, ou possuir redes relacionais inferiores a vinte pessoas). Ainda que possuam um registro situado no limiar da relevância estatística, os empregados de execução posicionam-se também nesta região do espaço social local. Em termos gerais e não obstante algumas especificidades decorrentes do maior protagonismo dos intelectuais, tudo indica, por isso, que estamos perante um processo de divisão social suscetível de ser conceitualizado no quadro de uma oposição em termos de volume global de capital possuído pelas famílias locais.

O Gráfico 2 diz respeito ao segundo eixo do espaço social local e apresenta uma configuração diferente da anterior. Esta acentua as associações entre os agentes que pertencem às frações técnicas e de enquadramento intermediário, que vivem em casas arrendadas, possuem doze anos de escolaridade e um número não muito denso, mas relativamente variado, de livros em casa e que possuem acesso à internet, conforme se verifica no lado superior da figura. Em alternativa, o lado inferior da mesma figura acentua a associação entre as pertenças às frações independentes e proprietárias da pequena-burguesia, as origens sociais camponesas, a propriedade de uma outra habitação, a escolaridade reduzida, a presença de alguns livros em casa, algum envolvimento associativo não acompanhado, contudo, pela ligação à internet. Num 
registro menos intenso, os empresários, dirigentes e profissionais liberais também contribuem para a polarização assim definida. O eixo do espaço social estabelecido deste modo acentua um efeito de composição local configurado em torno do capital técnico e um outro claramente estruturado em torno da propriedade econômica, sobretudo pequena.

GRÁFICO 2

Espaço Social do Bairro do Amial (Catorze Modalidades Ativas no Eixo 2)

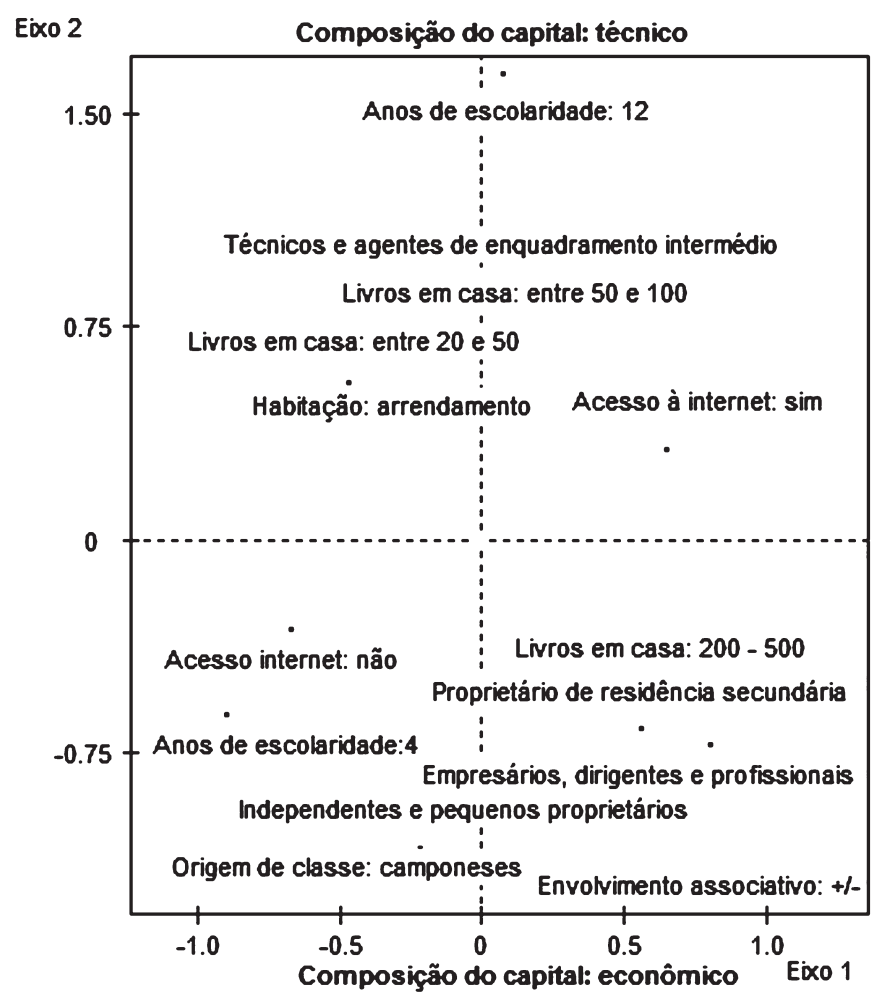

Fonte: PTDC/SDE/69996/2006, Inquérito IB18, 2009.

No Gráfico 3 mobilizamos informação sobre o sexo, a idade e a condição de trabalho dos residentes para caracterizar, em termos suplementares, a configuração do espaço social do bairro. As divisões em termos de volume global de capital identificadas no âmbito do primeiro eixo podem ser lidas a partir de divisões de sexo, etárias e de condição de trabalho: tendencialmente, os agentes com maior volume global de capital são homens, mais jovens, sendo também estudantes e economicamente ativos. As divisões relativas ao efeito de composição local dos capitais dos residentes locais são sobretudo caracterizadas por uma oposição definida pela idade: a relevância do capital técnico é uma característica dos jovens e adultos-jovens, enquanto a presença da pequena propriedade econômica é uma característica dos mais idosos. 
GRÁfICO 3

Projeção de Variáveis de Caracterização Social (Sexo, Grupo Etário e Condição Perante a Atividade Econômica) no Espaço Social do Bairro do Amial (Eixos 1 e 2)

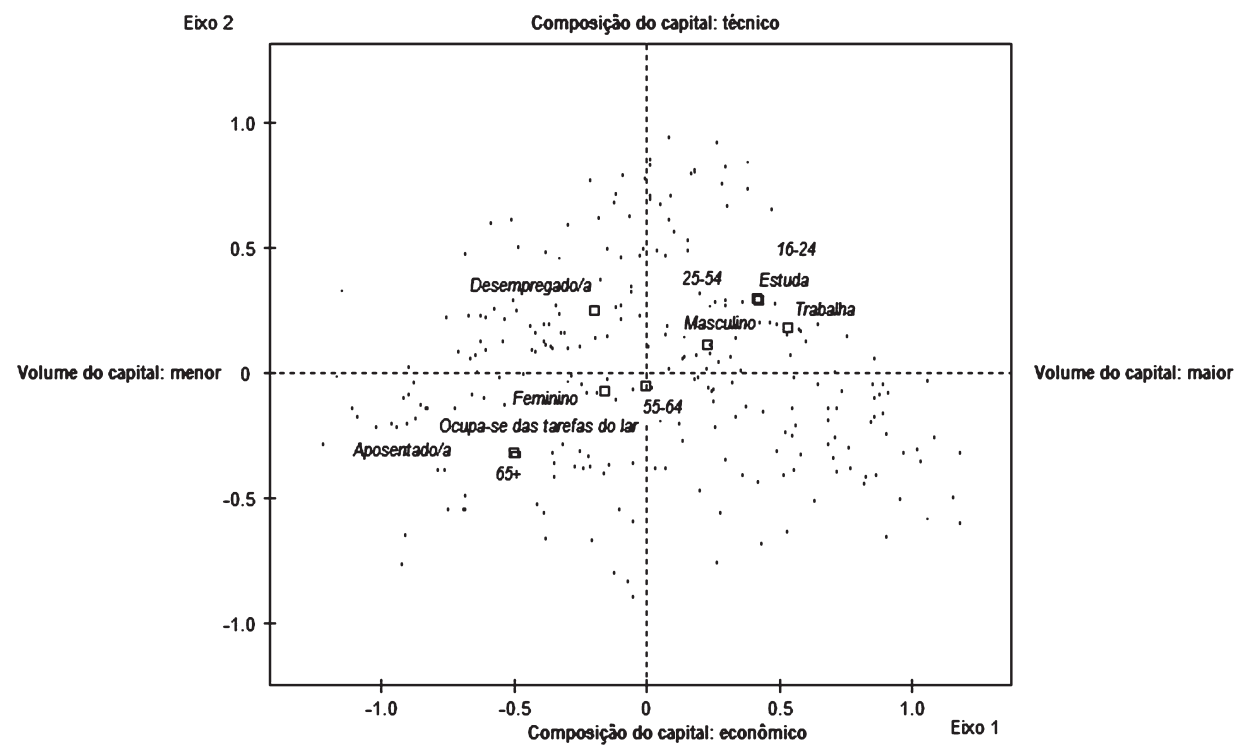

Fonte: PTDC/SDE/69996/2006, Inquérito IB 18, 2009.

A análise empreendida localmente contemplou ainda um inventário das vivências quotidianas dos residentes que contabilizou informação relativa a cerca de trinta variáveis sobre utilização do tempo e que foram lidas, globalmente, com base em três modalidades de intensidade distinta: menor (-), intermediária (+/-) e maior (+). Os Gráficos $4 \mathrm{e} 5$ apresentam informações sobre as modalidades das práticas consideradas no inquérito que podem ser significativamente mobilizadas para caracterizar cada um dos eixos previamente identificados no espaço social ao abrigo da utilização deste como mapa preditivo ${ }^{10}$. Conforme se pode verificar no Gráfico 4 , na região do espaço social local com maior volume global de capital (lado direito), podem encontrar-se, em termos suplementares, modalidades de práticas bem diferenciadas: com efeito, estas, para além do visionamento televisivo mais moderado (menos de duas horas por dia), da menor preponderância quotidiana das rotinas do tempo livre (realizar as tarefas domésticas ou ir às compras localmente) e do investimento sociabilitário doméstico (como receber pessoas em casa), relevam, fundamentalmente, a menor religiosidade e o desenvolvimento intenso, ou pelo menos relativamente regular, de práticas de consumo (ida ao centro comercial), de sociabilidade (ida ao café, jantar

10. Do ponto de vista técnico, a representação gráfica contemplou apenas modalidades com valor $t$ superior a 2,0 (Lebart et al., 1998). 
GRÁfICO 4

Projeção das Práticas Sociais dos Habitantes no Espaço Social do Bairro do Amial (Modalidades Suplementares com Valores $t>2.0$ no eixo 1)

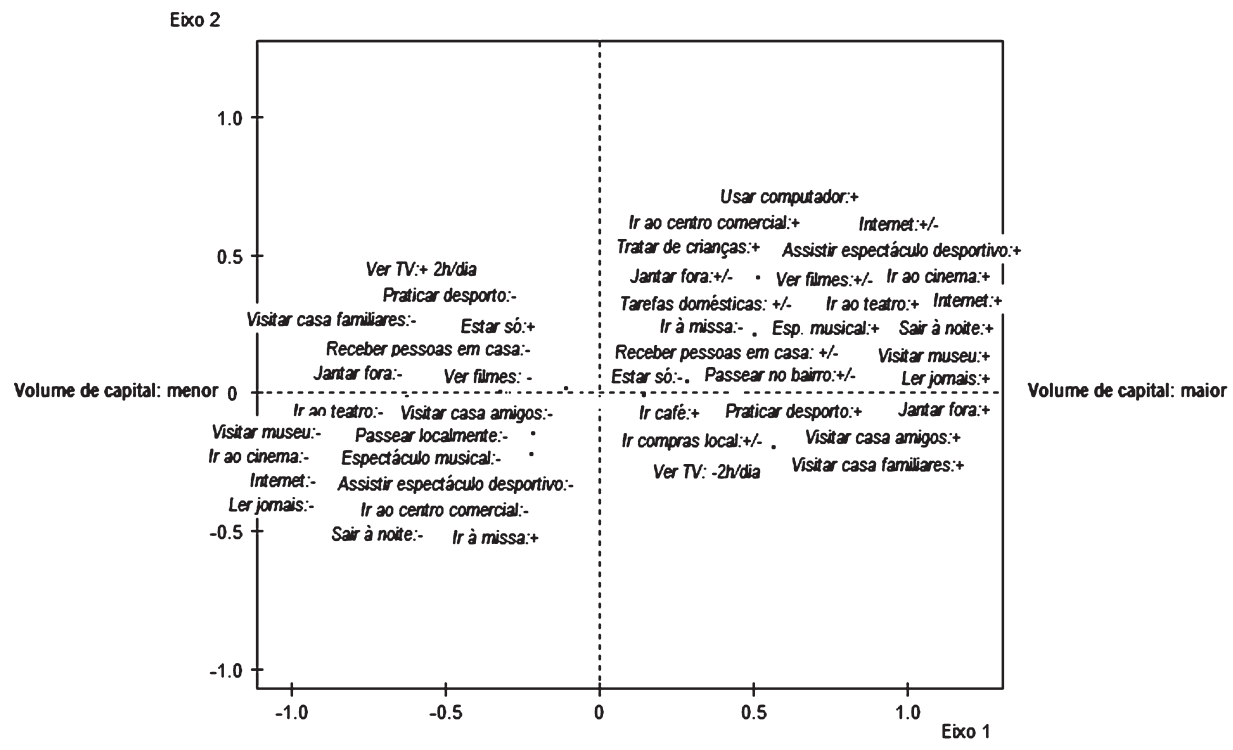

Fonte: PTDC/SDE/69996/2006, Inquérito IB 18, 2009.

\section{GRÁFICO 5}

Projeção das Práticas Sociais dos Habitantes no Espaço Social do Bairro do Amial (Modalidades Suplementares com Valores $t>2,0$ no Eixo 2)

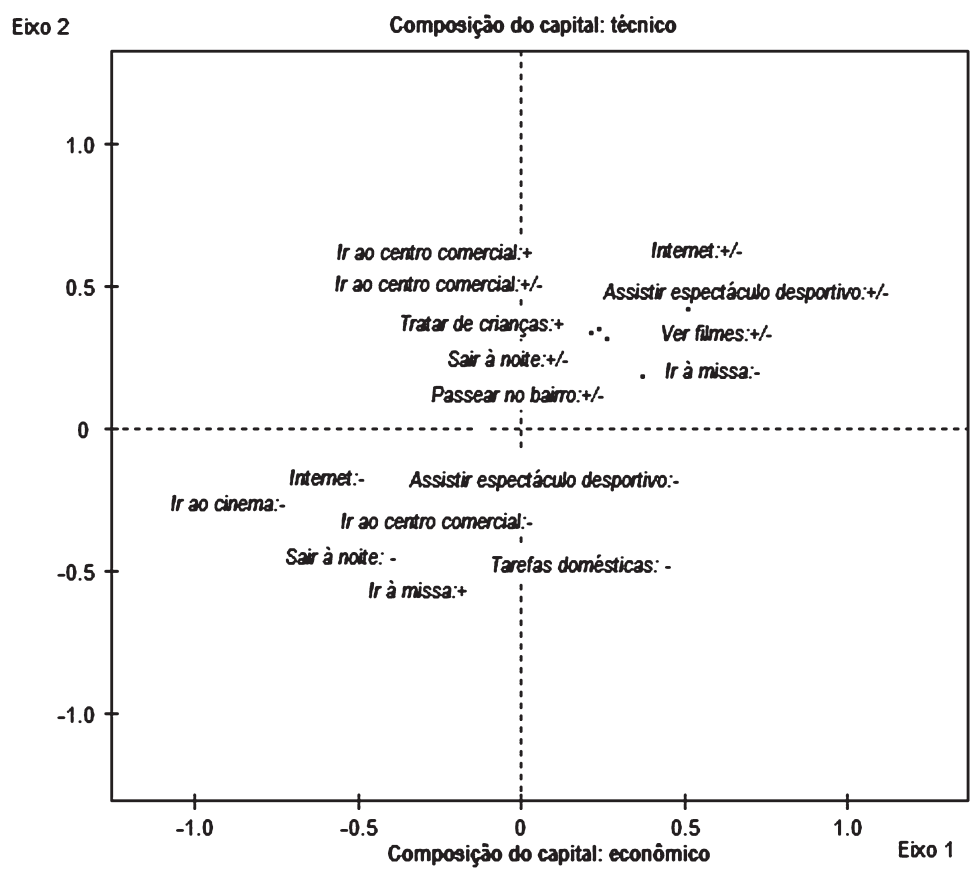

Fonte: PTDC/sDE/69996/2006, Inquérito IB 18, 2009. 
fora, visitar familiares e amigos), de orientação para a cultura (leitura muito regular de jornais e revistas, ir ao cinema, ao teatro, ou visitar museus), ou ainda de práticas desportivas. Quanto aos posicionamentos dotados de menor volume de capital (lado esquerdo) caracterizam-se, tal como se pode verificar no mesmo Gráfico 4, pela ausência de um conjunto muito significativo de práticas. Na realidade, aquelas que mais intensamente caracterizam o quotidiano desses agentes acentuam a importância da televisão (mais de duas horas por dia), mas também o relevo da solidão e das práticas religiosas regulares, como as que passam pela ida à missa.

No Gráfico 5 é possível identificar, em termos suplementares, as práticas sociais e culturais dos agentes que se posicionam no âmbito do segundo eixo do espaço social local. A região superior do eixo, definida pela importância do capital técnico, pode ser caracterizada por um conjunto de práticas sociais e culturais em que é possível destacar a preponderância do uso intenso do computador e da internet, do tempo ocupado com o cuidado de crianças, mas também a presença relativamente regular (ainda que menos intensa) de atividades religiosas, passeios locais pela vizinhança, saídas à noite, assistir a filmes, idas ao centro comercial e frequência de espetáculos desportivos. Em alternativa, a região inferior da mesma figura, configurada em torno da (pequena) propriedade econômica, assinala sobretudo tendências para um desenvolvimento menos regular de práticas determinadas: tarefas domésticas, uso do computador e da internet, saídas à noite, compras no centro comercial ou ida ao cinema constituem domínios de prática menos investidos. Os agentes que assim se posicionam caracterizam-se, sobretudo, pela grande importância da religiosidade católica, traduzida na ida muito regular à missa.

Não obstante a importância do processo de abandono de um segmento específico do edificado, que não deixa de se traduzir em alguma perda populacional e em envelhecimento, por comparação com a cidade modal, o Bairro do Amial tem sido marcado pelo reforço dos posicionamentos sociais e dos capitais econômicos e culturais de seus moradores. Uma tal tendência de qualificação, que acentua um traço de seletividade social inscrito no acesso ao bairro desde sua fundação, não impede, contudo, que se consolide no seu interior um quadro significativo de divisão social. O espaço social construído localmente permite identificar tal quadro. Com efeito, se o primeiro eixo de estruturação do espaço social configura uma oposição de volume global de capital, o segundo remete para o modo como localmente se estruturam os princípios da composição dos capitais, abrindo a análise ao estudo dos processos de diferenciação social no interior da pequena-burguesia. Assim, o primeiro dos eixos diferencia os agentes pertencentes às famílias mais qualificadas, em particular, os intelectuais (a fração de classe mais representada localmente), dos que possuem inserções operárias e de emprego de execução. $\mathrm{O}$ segundo eixo 
contrasta modalidades específicas de constituição de capital cultural e de capital econômico no interior das regiões intermediárias do espaço social local, relevando a divisão entre o capital técnico (Bourdieu, 2000, pp. 42-49) e a pequena propriedade econômica (Bourdieu, 1979, pp. 398-404). Dotados de uma morfologia social específica (o envelhecimento é mais sensível entre quem detém menor volume de capital e entre aqueles que possuem pequena propriedade econômica), os posicionamentos no espaço social assim estabelecidos caracterizam-se por estilos de vida diferenciados. A diversidade acumulada de práticas de sociabilidade e a participação cultural intensas encontram-se entre intelectuais e detentores de maior volume de capital, o que corrobora e permite afinar o retrato sobre a sua dinâmica constitutiva identificado previamente na cidade em geral; o quotidiano mais privatizado caracteriza operários e agentes com menor volume de capital e também é suscetível de ser conceptualizado à luz dos desenvolvimentos aferidos na cidade e noutros contextos societários (Schwartz, 1990; Beaud e Pialoux, 2003). Já os agentes mais dotados de capital técnico caracterizam-se por uma dinâmica de envolvimento sociabilitário nem sempre intensa, mas relativamente diversificada, com importância no desenvolvimento das relações de vizinhança locais; entre os pequenos proprietários destaca-se a propensão para um maior envolvimento religioso. Em suma, um lugar na cidade que, com frequência e por força da sua história e morfologia física, é pensado a partir de traços sociais mais simplificados envolve, na prática, e não obstante as forças sociais dominantes que o definem, a estruturação de posicionamentos sociais e de processos de simbolização diferenciados.

\section{Conclusão}

O corpo de proposições teóricas decorrente do programa de pesquisa de Bourdieu estabelecido em La distinction encontra respostas heurísticas no conjunto de trabalhos levado a cabo na cidade do Porto. As respostas documentadas, quer a propósito do espaço social, quer a propósito do espaço, dos estilos de vida parecem também, e por outro lado, encontrar complementaridades significativas com alguns dos estudos que, mais sistematicamente, têm assumido esses referenciais para dinamizar pesquisa empírica, seja a propósito de sociedades como a francesa, a norueguesa ou a dinamarquesa (Coulangeon e Lemel, 2009; Rosenlund, 2009; Prieur et al., 2008), seja como, ainda que mais parcialmente, a britânica (Bennett et al., 2009). Em complemento, o acionamento deste corpo de proposições teóricas e metodológicas no estudo dos bairros da cidade permite revelar as forças da física social em ação e as modalidades do seu encontro com os respetivos residentes. Os "efeitos de lugar" que aparecem no Bairro do Amial, analisado neste texto, permitem-nos conhecer 
melhor os posicionamentos sociais e os estilos de vida de seus residentes e a relação que estes estabelecem com as lógicas sociais e simbólicas que definem a cidade em geral.

\section{Referências Bibliográficas}

Almeida, João Ferreira de. (1986), Classes sociais nos campos. Lisboa, ICs.

Almeida, João Ferreira de et al. (1988), "Famílias, estudantes e universidade: painéis de observação sociográfica”. Sociologia, Problemas e Práticas, 4: 11-44.

. (2006), "Social classes and values in Europe". Portuguese Journal of Social Science, 2 (5): 95-117.

Beaud, Stéphane e Pialoux, Michel. (2003), Violences urbaines, violence sociale. Paris, Fayard. Bennett, Tony et al. (2009), Culture, class, distinction. Londres, Routledge.

BEnZÉCrI, Jean-Paul. (1992), Correspondence analysis handbook. Nova York, Marcel Dekker. Bourdieu, Pierre. (1966), “Différences et distinctions”. In: Bourdieu, Pierre e Darbel, Alain (orgs.). Le partage des bénéfices: expansion et inégalités en France. Paris, Minuit. . (1977), Algérie 60: structures économiques et structures temporelles. Paris, Minuit. . ([1978] 2013), "Symbolic capital and social classes". Journal of Classical Sociology, 2(13): 292-302. . (1979). La distinction: critique sociale du jugement. Paris, Minuit. - (1989), La noblesse d'État: grandes écoles et esprit de corps. Paris, Minuit. . (1992), Les règles de l'art: genèse et structure du champ littéraire. Paris, Seuil. . (1993a), "Esprits d'État: genèse et structure du champ bureaucratique". Actes de la Recherche en Sciences Sociales, 96: 49-62. . (1993b), “Comprendre". In: (org.). La misere du monde: la souffrance qui parle. Paris, Seuil, pp. 903-925. . (1993c), "Effets de lieu". In: (org.). La misère du monde: la souffrance quiparle.

Paris, Seuil, pp. 159-167.

. (1997), Méditations pascaliennes. Paris, Seuil.

. (2000), Les structures sociales de l'économie. Paris, Seuil. . (2003), "L'objectivation participante". Actes de la Recherche en Sciences Sociales, 150: $43-58$.

(2011), “Espaço social e génese das classes”. In: O poder simbólico. Lisboa, Edições 70, pp. 135-165.

Bourdieu, Pierre \& Saint-Martin, Monique de. (1976), "L'anatomie du gout". Actes de la Recherche en Sciences Sociales, 2: 2-112.

Bourdieu, Pierre \& SAYAD, Abdelmalek. (1964), Le déracinement: la crise de l'agriculture traditionnelle en Algérie. Paris, Minuit. 
Bourdieu, Pierre et al. (1963), Travail et travailleurs en Algérie. Paris/Haia, Mouton.

Bozon, Michel. (1984), Vie quotidienne et rapports sociaux dans une petite ville de province: la mise en scène des différences. Lyon, Presses Universitaires de Lyon.

Burawoy, Michael. (1998), "The extended case method”. Sociological Theory, 16: 4-33.

Coulangeon, Philippe \& Lemel, Yannick. (2009), "Les pratiques culturelles et sportives des Français: arbitrage, diversité et cumul". Economie et Statistique, 423: 3-30.

Donnat, Olivier. (1998), Les Pratiques culturelles des Français: enquête 1997. Paris, La Documentation Française.

Duval, Julien. (2010), "Distinction studies". Actes de la Recherche en Sciences Sociales, 181182: 147-156.

Elias, Norbert \& Dunning, Eric. (1992), A busca da excitação. Lisboa, Difel.

Lalive D'Epinay, Christian et al. (1982), Temps libre: culture de masse et cultures de classes aujourd'hui. Lausanne, Pierre-Marcel Favre.

LEBART, Ludovic et al. (1984), Multivariate descriptive statistical analysis: correspondence analysis and related techniques for large matrices. Nova York, John Wiley \& Sons.

Lebart, Ludovic et al. (1998), Statistique exploratoire multidimensionnelle. Paris, Dunod.

Le Roux, Brigitte \& Rounnet, Henry. (2004), Geometric data analysis. Dordrecht, Kluwer. . (2010), Multiple correspondence analysis. Londres, Sage.

Maget, Marcel. (1962), Guide d'étude directe des comportements culturels. Paris, CNRS.

Marques, Helder et al. (1990), Porto: percursos nos espaços e nas memórias. Porto, Afrontamento.

Martin-Criado, Enrique. (2008), Les deux Algéries de Pierre Bourdieu. Broissieux, Croquant.

Pereira, Virgílio Borges. (2005), Classes e culturas de classe das familias portuenses: classes sociais e modalidades de estilização da vida na cidade do Porto. Porto, Afrontamento.

. (2013), "L'espace social, les pratiques quotidiennes et la ville: repères pour une sociologie des divisions sociales et symboliques dans la ville de Porto". In: Coulangeon, Philippe \& Duval, Julien (orgs.) Trente ans après La distinction de Pierre Bourdieu. Paris, La Découverte, pp. 206-215.

. (2015), "The structuration of lifestyles in the city of Porto: a relational approach". In: HANQUinet, Laurie \& SAVAGE, Mike (orgs.). Routledge international handbook of sociology of art and culture. Londres, Routledge, pp. 421-435.

. (2016), "Urban distinctions: class, culture and sociability in the city of Porto". International Journal of Urban and Regional Research, special issue "Taking Bourdieu to Town”, no prelo.

Pereira, Virgílio Borges \& Pinto, José Madureira. (2012), "Espace, relations sociales et culture populaire dans le coeur ancien de la ville de Porto”. Sociétés Contemporaines, 86: 115-134.

Pereira, Virgílio Borges \& QueIrós, João. (2012a), “State, housing and the 'social question' in the city of Porto (1956-2006)”. Social Sciences, 1: 203-214.

. (2012b), Na modesta cidadezinha: génese e estruturação de um bairro de casas económicas do Porto (Amial, 1938-2010). Porto, Afrontamento. 
. (2013), “Une maison pour le 'peuple' portugais: genèse et trajectoire d'un quartier du programme des 'maisons économiques' à Porto (1938-1974)”. Politix, 101: 49-78.

. (2014), "It's not a bairro, is it?': subsistence sociability and focused avoidance in a public housing estate". Environment and Planning A, 6 (46): 1297-1316.

PInto, José Madureira. (1985), Estruturas sociais e práticas simbólico-ideológicas nos campos. Porto, Afrontamento.

PInto, Louis. (1998), Pierre Bourdieu et la théorie du monde sociale. Paris, Albin Michel.

Prieur, Annick et al. (2008), "Cultural capital today: a case study from Denmark". Poetics, 36: 45-70.

Rosenlund, Lennart. (2009), Exploring the city with Bourdieu: applying Pierre Bourdieu's theories and methods to study the community. Saarbrücken, VDM.

Rouanet, Henry et al. (2005), "A análise geométrica de questionários. A lição de La Distinction de Bourdieu”. Sociologia: Revista da Faculdade de Letras da Universidade do Porto, 15: 43-52.

Schwartz, Olivier. (1990), Le monde privé des ouvriers: hommes et femmes du nord. Paris, PUF.

Wacquant, Loïc (2004). “Following Pierre Bourdieu into the field”. Ethnography, 4 (5): 387-414.

. (2008), Urban outcasts: a comparative sociology of urban marginality. Cambridge, Polity.

. (2013), "Symbolic power and group-making: on Bourdieu's reframing of class". Journal of Classical Sociology, 2 (13): 274-291.

\section{Resumo}

Classes sociais e simbolização na cidade do Porto: elementos teóricos e resultados de pesquisa empírica

O presente texto procura demonstrar a relevância do trabalho de Pierre Bourdieu para o desenvolvimento de pesquisa sociológica sobre classes sociais e processos de simbolização na cidade. A partir de uma leitura de La distinction, o texto documenta o percurso teórico-empírico formulado na análise da cidade do Porto, em Portugal. Com recurso à técnica de análise de correspondências múltiplas, demonstra-se a relevância das perspectivas topológicas para o conhecimento das relações entre divisões sociais e simbólicas na cidade. Para esse efeito, elabora-se uma síntese interpretativa dos resultados das pesquisas efetuadas neste âmbito na cidade e analisa-se, mais pormenorizadamente, o modo como tais relações se estruturam num de seus bairros. Palavras-chave: Pierre Bourdieu; Classes sociais; Simbolização; Porto (Portugal). 


\section{Abstract}

Social classes and symbolization in the city of Porto: theoretical elements and empirical research results

This paper tries to demonstrate the relevance of Pierre Bourdieu's work for the development of sociological research about social classes and symbolic processes in the city. Based on a reading of La distinction, the paper documents the theoretical and empirical path that was formulated to analyse the city of Porto, in Portugal. With the help of multiple correspondence analysis, the paper shows the relevance of topological perspectives for the understanding of the relations between social and symbolic divisions in the city. For that purpose, the paper proposes an interpretative synthesis about the research results obtained in the city and analyses, with detail, the way these divisions are structured in one of the city's neighbourhoods.

Keywords: Pierre Bourdieu; Social classes; Symbolization; Porto (Portugal).

Texto enviado em 2/2/2016 e aprovado em 3/2/2016. DoI: 10.11606/0103-2070.ts.2016. 110722.

Virgílio Borges Pereira é professor do Departamento de Sociologia da Faculdade de Letras e Instituto de Sociologia da Universidade do Porto. E-mail: jpereira@letras.up.pt. 